\title{
Lactobacillus gasseri JM1 with potential probiotic characteristics alleviates inflammatory response by activating the PI3K/Akt signaling pathway in vitro
}

\author{
Linlin Sun, ${ }^{1 *}$ Wenli Tian, ${ }^{2 *}$ Xiaojie Guo, ${ }^{1}$ Yu Zhang, ${ }^{1}$ Xinyu Liu, ${ }^{1} \odot$ Xuesong Li, ${ }^{1} \odot$ Yazhen Tian, ${ }^{1}$ \\ Chaoxin Man, ${ }^{1} \dagger$ (D) and Yujun Jiang ${ }^{1} \dagger$ (1) \\ ${ }^{1}$ Key Laboratory of Dairy Science, Ministry of Education, Department of Food Science, Northeast Agricultural University, Harbin, 150030, China \\ ${ }^{2}$ Institute of Apicultural Research, Chinese Academy of Agricultural Sciences, Beijing 100093, P. R. China
}

\section{ABSTRACT}

Lactobacillus gasseri JM1, a novel strain isolated from infant feces, exhibited common probiotic properties such as high acid tolerance, bile salt tolerance, and adhesion to epithelial Caco-2 cells, suggesting its ability to survive in the gastrointestinal tract and confer potential probiotic action on the host. In the current study, we aimed to evaluate the immunomodulatory activity of $L$. gasseri JM1 and explore the underlying signaling pathways in vitro. The results showed that pretreatment with $L$. gasseri JM1 alleviated lipopolysaccharide-induced inflammatory response, as evidenced by downregulation of genes encoding proinflammatory cytokines $[I L 1 B, I L 6, I L 8$, and tumor necrosis factor- $\alpha(T N F A)]$ and upregulation of genes encoding anti-inflammatory cytokines $[I L 4, I L 10$, transforming growth factor- $\beta 3$ (TGFB3), and IFNG]. A high level of gene expression was noted for toll-like receptor 2 and NOD-like receptor 2. Meanwhile, transcriptomic sequencing obtained 84 differentially expressed genes. Kyoto Encyclopedia of Genes and Genomes analysis revealed the phosphatidylinositol 3-kinase (PI3K)/protein kinase B (Akt) signaling pathway was activated by L. gasseri JM1 in Caco-2 cells. Inhibitor of PI3K/ Akt played various roles in the release of cytokines, indicating that the pathway was involved in protecting the host against lipopolysaccharide stress. Moreover, whole-genome sequencing revealed fundamental genetic properties of L. gasseri JM1 and provided clues for probiotic characteristics. In summary, the strain could exert immunomodulatory effects via the toll-like receptor 2 and NOD2-mediated PI3K/Akt signaling pathway and be regarded as a potential probiotic.

Key words: Lactobacillus gasseri, probiotic characteristics, immunomodulatory activity, PI3K/Akt

\footnotetext{
Received January 11, 2020.

Accepted April 19, 2020.

*These authors contributed equally to this work.

†Corresponding authors: mcxwh2006@qq.com and yujun_jiang@ 163.com
}

\section{INTRODUCTION}

Lactobacillus gasseri, a well-characterized species, is generally regarded as an autochthonous microorganism in the gastrointestinal tract of humans and animals (Selle and Klaenhammer, 2013). Some strains of this species have been demonstrated to have beneficial effects, such as alleviation of allergic diseases (Martínez-Cañavate et al., 2009), anti-inflammatory properties (Niibo et al., 2019), regulation of oxidative stress (Kobatake et al., 2017), and inhibitory action against Helicobacter pylori (Sakamoto et al., 2001). Lactobacillus gasseri ATCC $33323^{\mathrm{T}}$, as the first strain to complete whole-genome sequencing, has been demonstrated to play an essential part in defense and protection of the human gastrointestinal tract (Azcarate-Peril et al., 2008). Hence, L. gasseri could be considered as a probiotic candidate.

Growing evidence has indicated that the intestinal microbiota is closely associated with host health (Sommer and Backhed, 2013; Costea et al., 2018), especially in immunoregulatory and anti-inflammatory effects. Lactobacillus spp., as potential probiotic microbes, can activate innate and adaptive immunity by colonizing the intestinal epithelial cells (Dongarrà et al., 2013). Innate immunity is characterized by the activation of immunocytes including natural killer cells, dendritic cells, and macrophages, which are able to phagocytize pathogenic bacteria and deliver antigens. Adaptive immunity consists of cellular and humoral immunity. Some lactobacilli confer immunoregulatory function on the host by releasing cytokines, which is deemed a significant role of probiotics (Marin et al., 1997). The proinflammatory cytokines IL-6, IL-12, and tumor necrosis factor (TNF)- $\alpha$ have been found to increase in intestinal epithelial cells under inflammatory conditions (Gao et al., 2017). Some reports proposed that antiinflammatory cytokines protect the intestinal barrier against damage and inhibit the occurrence of inflammation (Mirpuri et al., 2012), inclduing IL-4, IL-10, and transforming growth factor (TGF)- $\beta 3$. Pérez-Cano et al. (2010), using peripheral blood mononuclear cells 
stimulated with LPS, showed that innate and adaptive immunity responses were both enhanced, producing many cytokines and chemokines. Furthermore, some strains in $L$. gasseri are similarly recognized to possess immunoregulatory activity. Lactobacillus gasseri 4M13 has been demonstrated to play an effect in host immunity by modulating proinflammatory cytokines, whose potential probiotic properties were also revealed by whole-genome sequencing (Oh et al., 2018).

We previously demonstrated that Lactobacillus plantarum NDC 75017 and exopolysaccharides isolated from Lactobacillus acidophilus NCFM are involved in immunoregulatory effects by activating the NF- $\kappa \mathrm{B}$ and p38 mitogen-activated protein kinase pathways ( $\mathrm{Li}$ et al., 2014; Jiang et al., 2016). In addition, the study by Jang showed that the protein kinase B (Akt) signaling pathway might be associated with immunomodulation, such as the secretion of cytokines (Jang et al., 2013). Lactobacillus rhamnosus GG exhibited modulating effects on intestinal epithelia cells, leading to activation of phosphatidylinositol 3-kinase (PI3K)/Akt expression, as well as regulation of IL-8 (Huang and Huang, 2016). Thus, considering the specificity of strains, the probiotic characteristics of novel L. gasseri JM1 strain and underlying pathways involved in immunomodulatory activity are worth investigating further.

In this study, we evaluated immunomodulatory activity of L. gasseri JM1 with common probiotic properties on Caco-2 cells treated by LPS. By analyzing differentially expressed genes (DEG), the underlying signaling pathways involved in immunomodulatory activity in $L$. gasseri were predicted. Further, whole-genome sequencing was determined to gain comprehensive insight into genetic properties of JM1, which provided clues for potential probiotic characteristics.

\section{MATERIALS AND METHODS}

\section{Bacterial Strains and Cells}

Lactobacillus gasseri JM1 was originally isolated from a fecal sample collected from a 39-d-old breastfed healthy infant and spread on de Man, Rogosa, and Sharpe (MRS; Qingdao Hope Bio-Technology Co. Ltd., Qingdao City, China) agar plates. The strain was grown in MRS broth for $18 \mathrm{~h}$ at $37^{\circ} \mathrm{C}$ under anaerobic and static conditions, collected by centrifugation $(5,000$ $\times g$ at $4^{\circ} \mathrm{C}$ for $5 \mathrm{~min}$ ), and the pellet was suspended to $1.0 \times 10^{8}, 10^{6}$, and $10^{4} \mathrm{cfu} / \mathrm{mL}$ in Dulbecco's modified Eagle medium (DMEM, Beijing Solarbio Science \& Technology Co. Ltd., Beijing, China) for cell experiments. The pathogenic bacterium Escherichia coli ATCC 25922 was cultured in Luria-Bertani (LB; Qing- dao Hope Bio-Technology Co. Ltd.) broth at $37^{\circ} \mathrm{C}$ with shaking. The human adenocarcinoma colon cell line Caco-2 used in vitro anti-inflammatory activity was purchased from Shanghai Institutes for Biological Sciences, Chinese Academy of Sciences (Shanghai, China).

\section{Adhesion Assay}

Caco-2 cells were cultured in DMEM supplemented with $10 \%$ heat-inactivated fetal bovine serum, $1 \%$ nonessential amino acid and $1 \%$ penicillin-streptomycin solution at $37^{\circ} \mathrm{C}$ in $5 \% \mathrm{CO}_{2}$ incubator, as previously described ( $\mathrm{Li}$ et al., 2014). The culture medium was changed every second day and regularly checked to ensure normal morphology of cells. For the adhesion assay, Caco-2 cells were seeded at $1.0 \times 10^{6}$ cells/well in 6-well culture plates (Corning Inc., Corning, NY), then $1 \mathrm{~mL}$ of the $L$. gasseri suspension $\left(1.0 \times 10^{8} \mathrm{cfu} / \mathrm{mL}\right)$ was added to each well, which was incubated at $37^{\circ} \mathrm{C}$ in a $5 \% \mathrm{CO}_{2}$ atmosphere. After $2 \mathrm{~h}$ of incubation, the monolayers were washed thrice with $1 \mathrm{~mL}$ of PBS ( $\mathrm{pH}$ 7.4) and nonadherent cells were removed. Subsequently, the Caco-2 cells adhered by L. gasseri were digested with trypsin-EDTA and serially diluted. The number of viable cell-adhered L. gasseri was assessed by plating on MRS agar.

\section{Inhibitory Activity Against the Adhesion of E. coli to Caco-2 Cells}

The inhibition adhesion assays were performed as described by Ren et al. (2012) with a slight modification. Escherichia coli ATCC 25922 was cultured at $37^{\circ} \mathrm{C}$ overnight in LB broth and L. gasseri JM1 was grown in MRS at $37^{\circ} \mathrm{C}$ for $18 \mathrm{~h}$. Before adhesion, strains were suspended to $1.0 \times 10^{8} \mathrm{cfu} / \mathrm{mL}$ in the DMEM. Caco-2 monolayers seeded in 6-well culture plates were washed 3 times with PBS. The assays were divided into 4 groups: individual group treated with $1 \mathrm{~mL}$ of E. coli alone, protection group treated with $500 \mu \mathrm{L}$ of L. gasseri and $500 \mu \mathrm{L}$ of $E$. coli in order, competition group inoculated with $1 \mathrm{~mL}$ of mixture containing $L$. gasseri and E. coli, and displacement group cocultured with $500 \mu \mathrm{L}$ of $E$. coli and $500 \mu \mathrm{L}$ of L. gasseri in order. The plates were incubated at $37^{\circ} \mathrm{C}$ in the $5 \% \mathrm{CO}_{2}$ incubator for $2 \mathrm{~h}$, after which the nonadherent cells were discarded and cell lysates were serially diluted as described above. The colony counts of E. coli ATCC 25922 that adhered to Caco-2 cells were tested through plating on LB agar. Percent inhibition was calculated by the percentage reduction of pathogenic bacteria adhering to Caco-2 cells when compared with the groups treated with L. gasseri JM1. 


\section{Immunoregulatory Activity of L. gasseri JM1 on Caco-2 Cells Stimulated by LPS}

To evaluate the anti-inflammatory activity, Caco-2 cells were stimulated in the absence of $L$. gasseri JM1 $\left(1.0 \times 10^{8}, 10^{6}\right.$, and $\left.10^{4} \mathrm{cfu} / \mathrm{mL}\right)$ with LPS $(1 \mu \mathrm{g} / \mathrm{mL})$, which was purified from E. coli (Sigma, St. Louis, MO). The Caco-2 cells treated with DMEM only were considered as the negative control. When the adherent cells reached polarizable status fully, the penicillin-streptomycin in culture medium was removed. The Caco-2 cells along with DMEM containing L. gasseri were incubated for $6 \mathrm{~h}$. Then, the bacteria in the medium were withdrawn from 6-well culture plates. An LPS solution was added to stimulate cells and incubated for another $2 \mathrm{~h}$. After incubation, the monolayers were washed 3 times with sterile PBS before RNA extraction. The RNA was collected in an RNA-free tube and stored at $-80^{\circ} \mathrm{C}$ for further cytokine assays.

\section{Inhibitor Treatment in Caco-2 Cells}

To investigate whether the PI3K/Akt pathway took part in the regulation of immunoregulatory activity, inhibitor treatment assay was carried out. Monolayers of Caco-2 cells, which reached polarization fully, were pretreated with $20 \mu M$ LY294002 (PI3K/Akt inhibitor) for $30 \mathrm{~min}$, and then incubated with L. gasseri JM1 and LPS. The cells were washed thrice before extracting RNA, and cells untreated with inhibitor were cognized as the control group.

\section{Real-Time Reverse-Transcription PCR Assay}

The RNA extracted from Caco-2 cells was reversed transcribed into cDNA using the PrimeScript RT reagent kit, according to the manufacturer's protocol (Dang et al., 2018). To investigate the expression of target genes, anti-inflammatory cytokines (IL-4, IL-10, IFN- $\gamma$, and TGF- $\beta 3$ ), proinflammatory cytokines (IL1 $\beta$, IL-6, IL-8, and TNF- $\alpha$ ), toll-like receptors (TLR1, TLR2, TLR4, and TLR6), and NOD-like receptors (NOD1 and NOD2) were determined by the QuantStudio 3 Real-Time PCR system (Applied Biosystems, Foster City) with TB Green Premix Ex Taq II (TaKaRa, Dalian, China). The $2^{-\Delta \Delta \mathrm{Ct}}$ method was used to calculate the relative expression of target genes, with $G A P D H$ serving as an endogenous gene. The sequences of primers are listed in Table 1.

\section{Transcriptomic Analysis}

RNA Extraction and Library Construction. Total RNA of Caco-2 cells incubated with L. gasseri or DMEM for $6 \mathrm{~h}$ was extracted using a kit following the manufacturer's instructions (Code No. BSC52M1, Bioer Technology, Hangzhou, China). The integrity of RNA was measured by electrophoresis on 1\% agarose gels and the Agilent 2100 Liquid Chromatogram (Agilent Technologies, Santa Clara, CA). The purity and concentration were examined by Nanodrop 2000 (Thermo Fisher Scientific, Waltham, MA). When all of the indicators met the criteria, oligo dT magnetic beads were used to enrich polyA and the purified mRNA was interrupted into small fragments of $300 \mathrm{bp}$. With mRNA fragments serving as template, the first-strand cDNA was synthesized with random N6 primers and reverse transcriptase. Then, the second-strand $\mathrm{cDNA}$ was also synthesized by adding dNTP and DNA polymerase I. After the library fragments were constructed, PCR amplification was carried out to select cDNA fragments of $450 \mathrm{bp}$ in length, and the quality of the library was evaluated using Agilent Bioanalyzer 2100 system. Based on the effective concentration and the amount of data, the libraries containing different index sequences were mixed according to the proportion, which were diluted and denatured to generate a single-strand library. Finally, the Illumina Hiseq Xten (Illumina, San Diego, CA) platform was performed to analyze the paired ends and raw data, following the standard manufacturer's protocol.

Identification of $\boldsymbol{D E G}$. Raw data were filtered first and clean data were aligned to the homo genome through Hisat2. To assess the gene expression in 2 groups, we used the fragments per kilo bases per million fragments (FPKM) method to normalized the read count. The DEG were identified utilizing DESeq software (version 1.30.0), with the conditions of $\mid \log _{2}$ fold change $\mid>1$ and $P$-value $<0.05$. In addition, Gene Ontology (GO) enrichment analysis was performed to analyze the biological function of DEG, and Kyoto Encyclopedia of Genes and Genomes (KEGG) was carried out to predict the pathway enriched by DEG. The topGO package (version 3.10) was applied for testing GO terms and the analysis of KEGG pathways was carried out using the hypergeometric distribution algorithm (version 3.6.2; Kanehisa et al., 2004; Powell et al., 2012). According to the results of KEGG, the potential pathways associated with immunoregulatory activity in L. gasseri JM1 were researched further.

\section{Whole-Genome Sequencing}

DNA Extraction and Sequencing. Genomic DNA was extracted from the harvested cells of $L$. gasseri JM1 using a Bacteria DNA kit (Beijing Tiangen Biotech Co. Ltd., Beijing, China) according to the instructions as mentioned in the manual. The quality of DNA 
was checked using agarose gel electrophoresis and the concentration was quantified by Qubit 2.0 Fluorometer (Thermo Fisher Scientific). Meanwhile, whole-genome sequencing of $L$. gasseri JM1 was obtained using the method of Whole Genome Shotgun with PacBio Sequel platform (Illumina Inc.) and Illumina NovaSeq (Pacific Biosciences, Menlo Park, CA) Two sequencing libraries with an average insert size of $10 \mathrm{~kb}$ and $350 \mathrm{bp}$ were constructed and raw data with low quality were filtered. The de novo genome was assembled using SMRT Link software version 5.1.0 (Ardui et al., 2018). To confirm accuracy, the assembly was optimized by arrow software (https://www.pacb.com/support/ software-downloads/) to distinguish error matching and corrected by second generation sequencing. Then, genome components such as transfer RNA and ribosomal RNA were predicted by tRNAscan-S (version 1.3.1; http://lowelab.ucsc.edu/tRNAscan-SE/index .html) and rRNAmmer software (version 1.2; http:// www.cbs.dtu.dk/services/RNAmmer/), respectively. The presence of CRISPR was identified by the CRISPR digger tool (https://crispr.i2bc.paris-saclay.fr/Server/) and the IslandPath-DIOMB program (https://github .com/brinkmanlab/islandpath) was used to search for genomic islands. Functional annotation of coding genes was implemented using the Cluster of Orthologous Groups (COG) of proteins provided by the National Center for Biotechnology Information (Galperin et al., 2015). In addition, the visualization of chromosome and plasmid were drawn using Circos (Krzywinski et al., 2009).

Comparative Genomics. To obtain the genomic translocation and inversion, the synteny analysis was carried out using Mauve software (version 2.4.0; http: //asap.genetics.wisc.edu/software/mauve/download .php). In addition, the sequences of 4 reference strains including L. gasseri ATCC $33323^{\mathrm{T}}$ (GenBank accession number NC_008530.1), DSM 14869 (GenBank accession number NZ_CP006803.1), 4M13 (GenBank accession number NZ_CP021427.1), and K7 (GenBank accession number NZ_KL402718.1) were downloaded from the National Center for Biotechnology Information and used for the analysis of core-pan genes by BPGA software (version 1.3; https://sourceforge.net/ projects/bpgatool/). The phylogenetic tree was built using MEGA (version 7.0; http://www.megasoftware

Table 1. Primer sequences for cytokines and receptors by real-time reverse-transcription PCR

\begin{tabular}{|c|c|}
\hline Gene & Primer sequence $\left(5^{\prime} \rightarrow 3^{\prime}\right)^{1}$ \\
\hline \multicolumn{2}{|c|}{ Housekeeping gene } \\
\hline \multirow[t]{2}{*}{$G A P D H$} & F: AACGGATTTGGTCGTATTG \\
\hline & R: GCTCCTGGAAGATGGTGAT \\
\hline \multicolumn{2}{|c|}{ Proinflammatory cytokine } \\
\hline \multirow[t]{2}{*}{$I L 1 B$} & F: GTGGCAATGAGGATGACTTGTTC \\
\hline & R: TTGCTGTAGTGGTCGGAG \\
\hline \multirow[t]{2}{*}{ IL 6} & F: GAGAAAGGAGACATGTAACAAGAG \\
\hline & R: GCTCTGGCTTGTTCCTCAC \\
\hline \multirow[t]{2}{*}{$I L 8$} & F: TCCAAACCTTTCCACCC \\
\hline & R: АACTTCTCCACAАСССТCTG \\
\hline \multirow[t]{2}{*}{ TNFA } & F: GGCAGTCAGATCATCTTCTCGAAC \\
\hline & R: TGGTAGGAGACGGCGATGC \\
\hline \multicolumn{2}{|c|}{ Anti-inflammatory cytokine } \\
\hline \multirow[t]{2}{*}{$I L 4$} & F: TCATTTTCCCTCGGTTTCAG \\
\hline & R: AGAACAGAGGGGGAAGCAGT \\
\hline \multirow[t]{2}{*}{ IL10 } & F: TCAGGGTGGCGACTCTAT \\
\hline & R: TGGGCTTCTTCTAAATCGTTC \\
\hline \multirow[t]{2}{*}{$I F N G$} & F: ATATCTTGGCTTTTCAGCTC \\
\hline & R: CTCCTTTTTCGCTTCCCTGT \\
\hline \multirow[t]{2}{*}{ TGFB3 } & F: CTGAGAATCACGGTGGTAAA \\
\hline & R: CATCTCAACTTACCATCCCT \\
\hline \multicolumn{2}{|l|}{ Receptor } \\
\hline \multirow[t]{2}{*}{ TLR1 } & F: GGGCACCCCTACAAAAGGAA \\
\hline & R: TGGCAAAATGGAAGATGCTAGTC \\
\hline \multirow[t]{2}{*}{$T L R 2$} & F: GATGCCTACTGGGTGGAG \\
\hline & R: AAAGACGGAAATGGGAGA \\
\hline \multirow[t]{2}{*}{$T L R 4$} & F: GGAGACTTGGCCCTAAACCA \\
\hline & R: AAGAAGCAATGGAATCGGGGT \\
\hline \multirow[t]{2}{*}{ TLR6 } & F: TCTTGGGATTGAGTGCTA \\
\hline & R: TCTTGGGATTGAGTGCTA \\
\hline \multirow[t]{2}{*}{ NOD1 } & F: ACAGAGCAACACTGGCATAA \\
\hline & R: TCCGCTTCTCATCTTCATAG \\
\hline \multirow[t]{2}{*}{ NOD2 } & F: TAACCTTTGATGGCTTTGACG \\
\hline & R: CACCTTGCGGGCATTCTT \\
\hline
\end{tabular}


.net/) based on single-copy core genes, which exhibited the evolutionary relationship and topology structure.

\section{Statistical Analysis}

All of the association assays were carried out in triplicate, and results were expressed as the mean \pm standard deviation. The Origin Pro 9.1 software (OriginLab Inc., Northampton, MA) was used for drawing. Statistical significance analysis was performed with the oneway ANOVA and Student-Newman-Keuls test using the SPSS 17.0 software (SPSS Inc., Chicago, IL), with values of $P<0.05$ regarding as statistically significant.

\section{RESULTS}

\section{Inhibition of L. gasseri in the Adhesion of E. coli to Caco-2 Cells}

In the present study, Caco- 2 cells as an in vitro model were used to assess adhesion capacity of $L$. gasseri JM1. Compared with E. coli ATCC 25922, the strain showed a better capacity of adhesion to Caco- 2 cells, with 7.56 $\log \mathrm{cfu} / \mathrm{mL}$ (Figure 1A). The inhibition rate of adhesion reached $12.4 \%$ in the competition assay, suggesting L. gasseri could prevent pathogenic bacteria from adhering to Caco-2 cells. However, inhibition activity decreased nonsignificantly when $L$. gasseri was not added to monolayers together with $E$. coli, with 7.39 and $7.50 \log \mathrm{cfu} / \mathrm{mL}$ adhering to cells in protection and displacement groups, respectively (Figure 1B).

\section{Anti-Inflammatory Effects of L. gasseri JM1 on LPS-Stimulated Cells}

The expression of proinflammatory and anti-inflammatory cytokines was investigated to evaluate inflam-

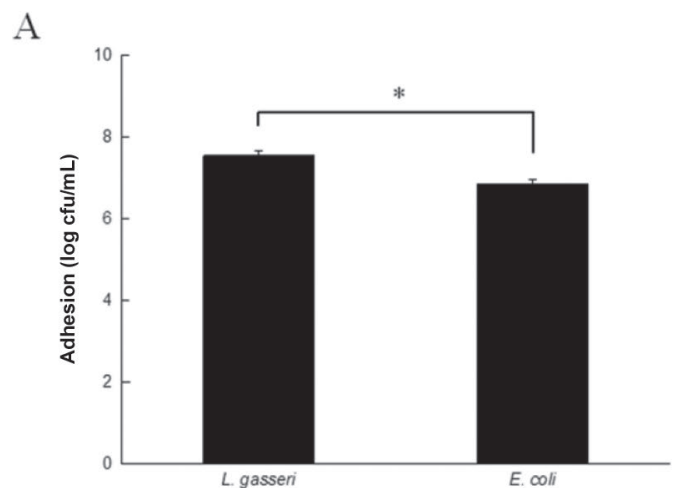

matory effects of $L$. gasseri JM1 on LPS-stimulated cells (Figure 2). Compared with the negative control group, Caco-2 cells had an induced inflammatory response in the LPS-stimulated group and the expression of proinflammatory cytokine genes, such as $I L 1 B$, IL6, IL8, and TNFA, were significantly increased $(P<$ $0.05)$. However, when Caco-2 cells were pretreated with $10^{8}$ and $10^{6} \mathrm{cfu} / \mathrm{mL}$ of $L$. gasseri, gene expression of these cytokines was downregulated significantly $(P<$ 0.05). Among all the proinflammatory cytokine genes, we found the suppressed action of the isolate on $I L 8$ was the most obvious. In contrast, significant upregulation of mRNA expression levels was obtained for other anti-inflammatory cytokines (IL4, IL10, TGFB3, and IFNG; $P<0.05$ ). It reached a maximum level for $I L 4$, indicating the anti-inflammatory potential of $L$. gasseri JM1 under LPS conditions.

\section{Effect of L. gasseri JM1 on the Expression of Toll-Like Receptors and NOD-Like Receptors}

Toll-like receptors (TLR) and NOD-like receptors (NLR) have been regarded as the important pattern recognition receptors (PRR), which exist in cytomembrane and cytoplasm, respectively, and activate signaling pathways by recognizing pathogen-associated molecular patterns such as peptidoglycan, formylated peptide, and LPS (Yamamoto and Akira, 2005; Zhang et al., 2008). To this end, we focused on investigating the expression of TLR and NLR genes in Caco-2 cells incubated with $L$. gasseri for $6 \mathrm{~h}$ (Figure 3). In the TLR studied, TLR2 and TLR6 showed the significant upregulation compared with the normal control group $(P<0.01)$. However, negligible changes were observed for TLR4. On the other hand, significantly higher expression of NOD2 was observed $(P<0.01)$ and NOD1 showed no significant expression in L. gasseri-treated

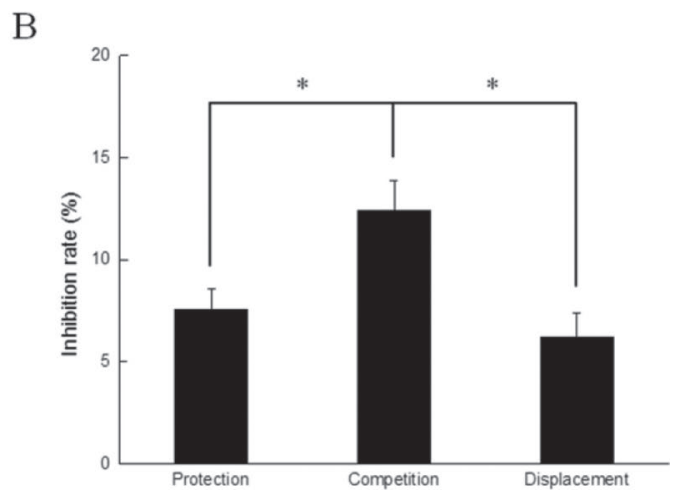

Figure 1. Adhesion effects of Lactobacillus gasseri JM1 and Escherichia coli ATCC 25922 on Caco-2 cells (A). Inhibition effects of L. gasseri JM1 in the adhesion of $E$. coli ATCC 25922 to Caco-2 cells (B). Data are presented as mean \pm SD in triplicate. ${ }^{*} P<0.05$ was regarded as statistically significant. 
A
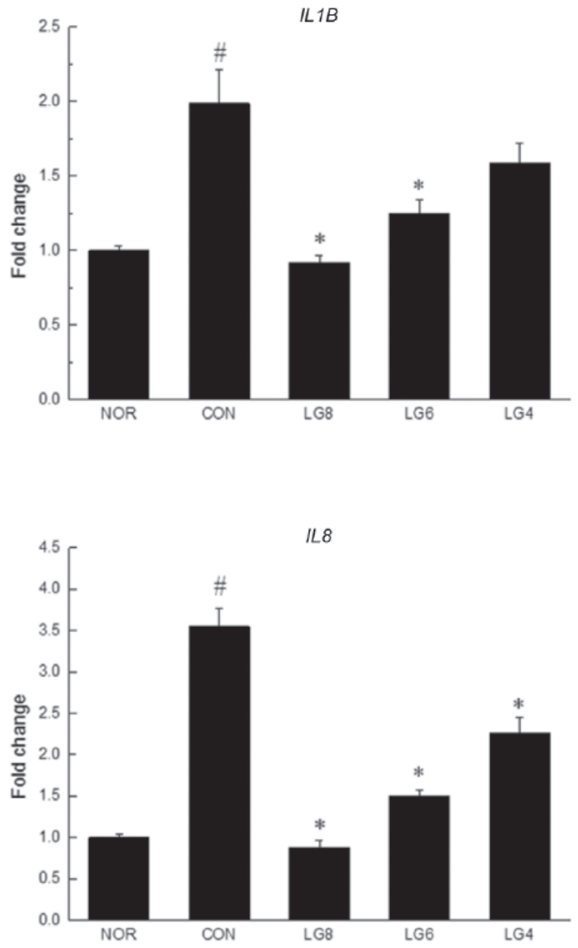

$\mathrm{B}$
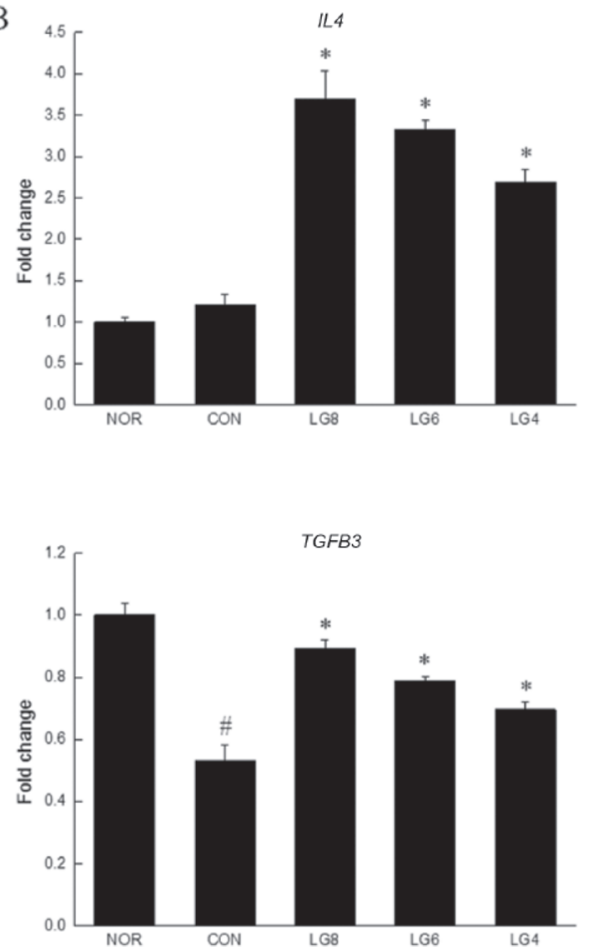
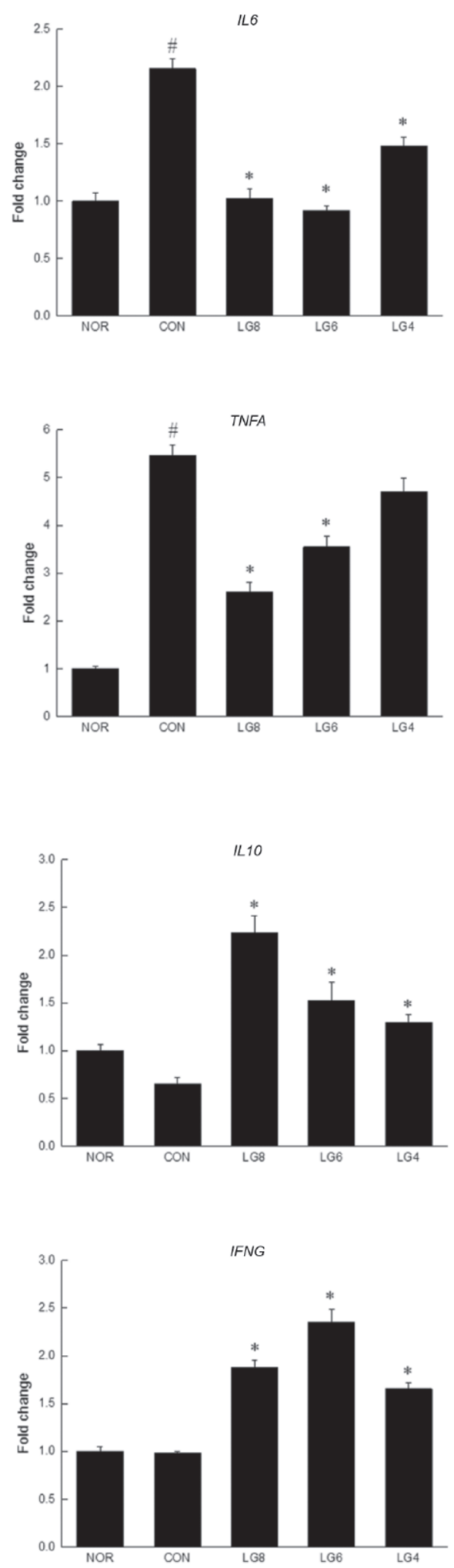

Figure 2. The mRNA expression of proinflammatory cytokines (A) and anti-inflammatory cytokines (B) in LPS-stimulated Caco-2 cells using real-time reverse-transcription PCR. Normal control group (NOR) was treated with Dulbecco's modified Eagle medium (DMEM) alone for 8 h. Caco-2 cells were pretreated with DMEM (CON) or Lactobacillus gasseri JM1 (LG8, $1.0 \times 10^{8} \mathrm{cfu} / \mathrm{mL} ; \mathrm{LG} 6,1.0 \times 10^{6} \mathrm{cfu} / \mathrm{mL} ; \mathrm{LG} 4,1.0$ $\times 10^{4} \mathrm{cfu} / \mathrm{mL}$ ) for $6 \mathrm{~h}$ and then stimulated with LPS for another $2 \mathrm{~h}$. Data are presented as mean $\pm \mathrm{SD}$ in triplicate. \#P<0.05, significantly different versus NOR. ${ }^{*} P<0.05$, significantly different versus CON. TNF $=$ tumor necrosis factor; TGF $=$ transforming growth factor. 
Caco-2 cells. Some reports have claimed that NOD receptor expression not only plays important roles in intestinal immunity but also has a relationship with insulin resistance, allergic diseases, and tumorigenesis (Kobayashi et al., 2005).

\section{Transcriptomic Sequencing and Bioinformatics Analysis}

After evaluating the effect of L. gasseri JM1 on the production of cytokines, transcriptomic sequencing was used to predict the underlying pathways (SRA accession number PRJNA615793). Differentially expressed genes at the mRNA level were investigated by comparing transcriptomic response of Caco-2 cells treated with L. gasseri JM1 or DMEM for 6 h. Bioinformatics analysis showed a total of 84 genes were differentially expressed with statistical significance $(P<0.05)$, of which 72 were upregulated and 12 were downregulated. Furthermore, GO enrichment assigned 84 DEG to 3 categories, covering biological process, cellular process, and molecular function. The top 10 terms of every category are exhibited in Figure 4A. According to the results of KEGG analysis, the DEG were enriched in such immunoregulatory pathways as PI3K/Akt and Jak-STAT. The bubble diagram represented the top 20 pathways with the most significant enrichment (Figure 4B).

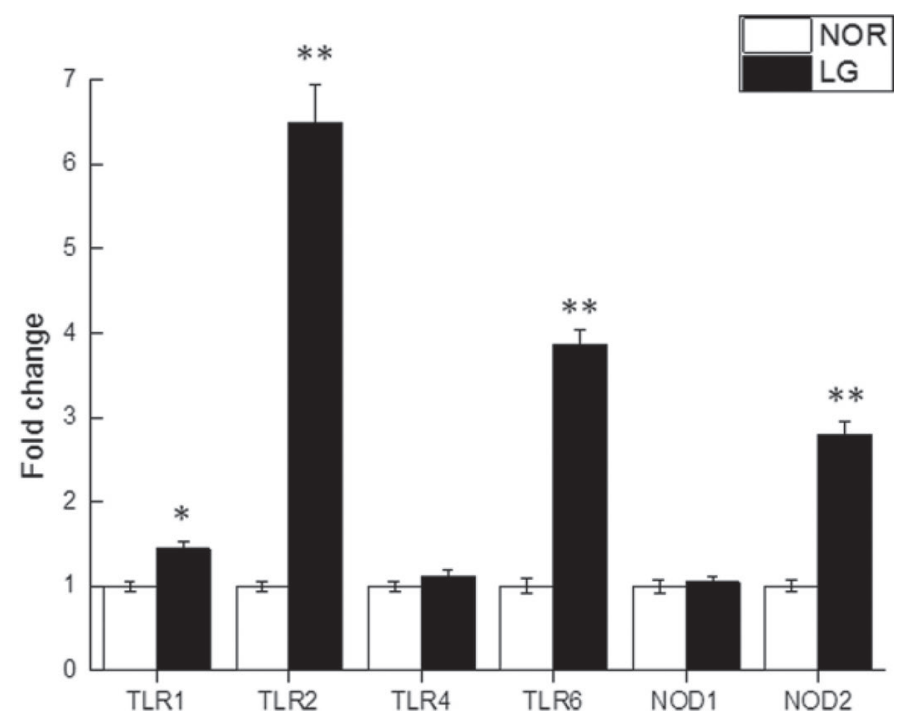

Figure 3. Effect of Lactobacillus gasseri JM1 on the expression of toll-like receptors (TLR) and NOD-like receptors (NOD) in Caco-2 cells. Cells were incubated with Dulbecco's modified Eagle medium only (NOR) or $1.0 \times 10^{8} \mathrm{cfu} / \mathrm{mL}$ of L. gasseri JM1 (LG) for $6 \mathrm{~h}$. The expression levels of TLR1, TLR2, TLR4, TLR6, NOD1, and NOD2 were determined by real-time reverse-transcription PCR. Data are presented as mean $\pm \mathrm{SD}$ in triplicate. ${ }^{*} P<0.05,{ }^{*} P<0.01$ compared with NOR.
To verify the results of transcriptomic sequencing, we selected 10 DEG associated with immunomodulation and determined the fold change using real-time reversetranscription PCR. Figure 4C showed that the tendency of genes measured by real-time reverse-transcription PCR was consistent with sequencing, suggesting the reliability and accuracy of transcriptomic sequencing in this study.

\section{Inhibition of PI3K/Akt Pathway in LPS-Stimulated Caco-2 Cells}

Activating TLR and NLR by Lactobacillus, host cells were induced to produce cytokines and chemokines via immunoregulatory pathways (Yamamoto and Akira, 2005; Lim et al., 2009). As shown in Figure 5, expression of some genes (IL1B,IL6,IL8,IL10, and TNFA) was considerably enhanced and others (IL4 and IFNG) were found to be suppressed in the presence of PI3K/ Akt inhibitor $(P<0.05)$, and no significant effect on TGFB3 was observed. This indicated the PI3K/Akt pathway did play an important role in the release of cytokines. In summary, these results revealed that $L$. gasseri JM1 may activate the PI3K/Akt signaling pathway to take part in immunoregulation by promoting the expression of TLR2 and NOD2. Additionally, given the complexity of immunoregulatory effects in the human intestine, comprehensive mechanisms between PI3K/ Akt pathway and cytokines still require further study.

\section{General Features of the Genome}

The whole genome of L. gasseri JM1 consisted of a 1,996,913-bp circular chromosome and 2 circular plasmids with lengths of 44,217 and 44,285 bp (GenBank accession number CP044412-CP044414; Figure 6A). The chromosome was predicted to encode 2,050 genes with a GC content of $34.93 \%$, whereas 2 plasmids contained 112 protein-coding genes. The genome contained 73 predicted transfer RNA genes. Fifteen ribosomal RNA gene operons, 3 CRISPR, and 9 genomic islands were found. Among the 2,162 identified coding genes, 1,250 genes were classified into 22 functional categories against the COG database (Figure 6B). It was notable that L. gasseri JM1 encoded the highest number of proteins that were associated with "Translation, ribosomal structure and biogenesis $(\mathrm{J})$," followed by "Carbohydrate transport and metabolism $(\mathrm{G})$ " and "Amino acid transport and metabolism (E)," and 69 coding genes were identified as "Function unknown (S)." Furthermore, the L. gasseri JM1 genome revealed some genes known to be involved in acid and bile salt stress. The strain contained 6 genes related to acid stress; 3 of them encoded F0F1-ATPase (JM1_GM001395, 


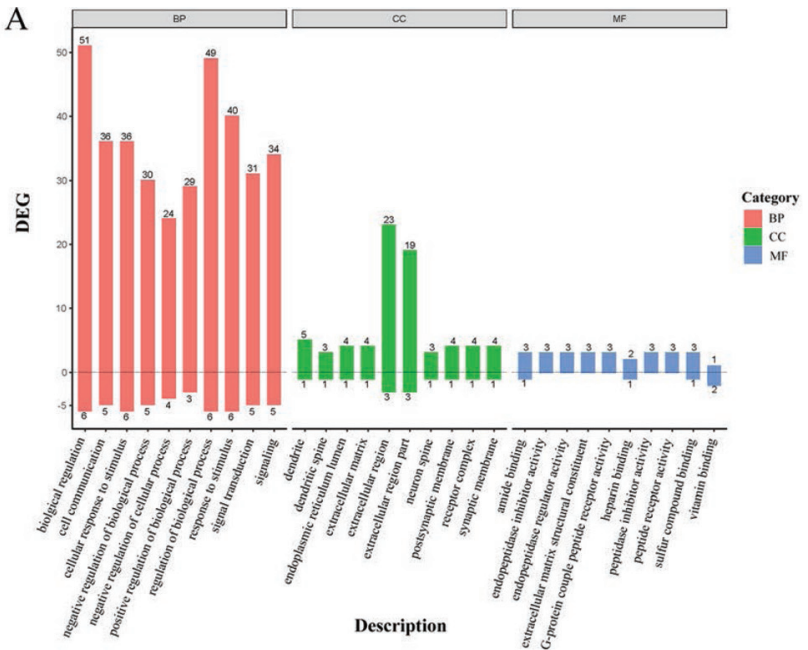

B
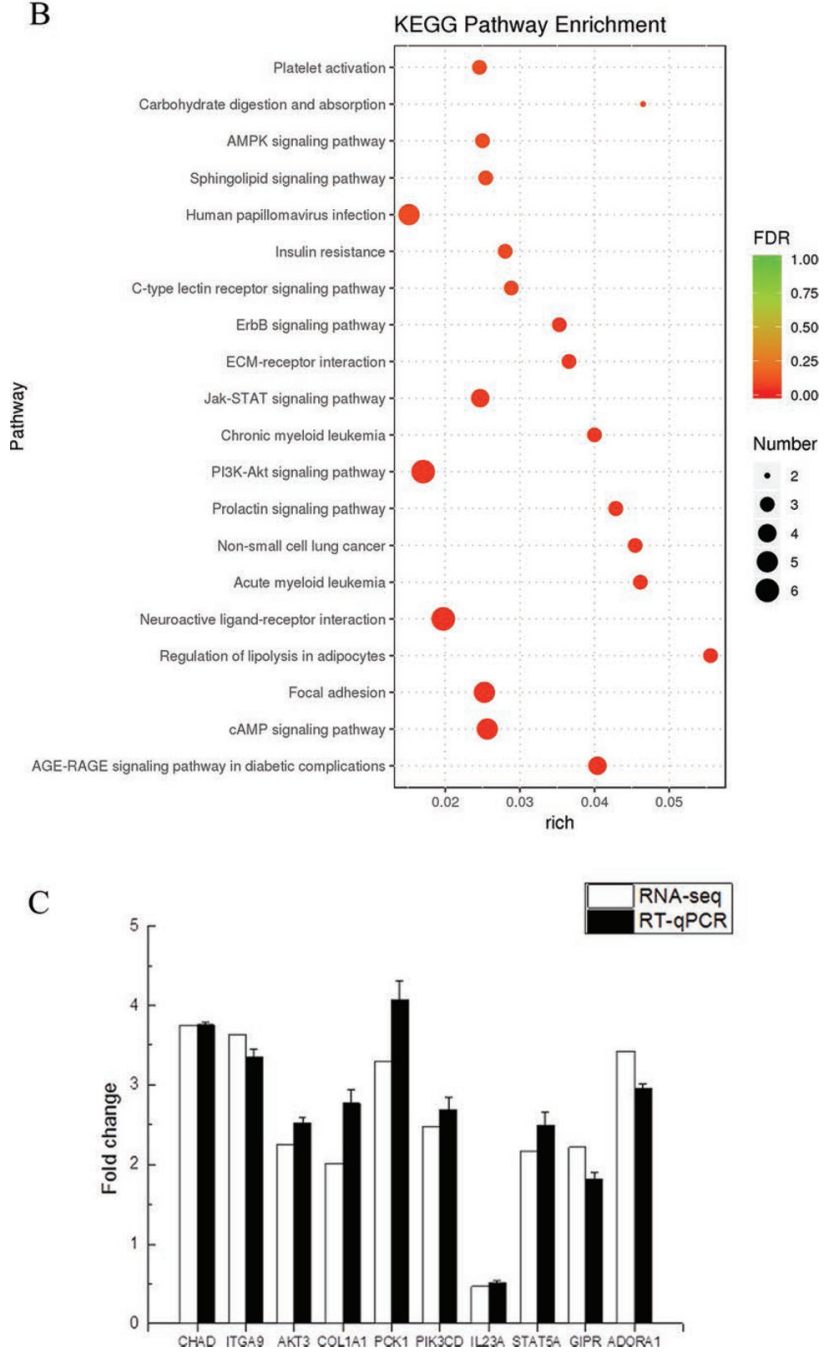

Figure 4. Transcriptomic sequencing analysis and verification in Lactobacillus gasseri JM1-stimulated Caco-2 cells. Gene Ontology function classification of upregulated and downregulated differentially expressed genes (DEG) between the control group and experimental group (A). The top 20 pathways enriched by DEG based on Kyoto Encyclopedia of Genes and Genomes (KEGG) analysis (B). The size of the dot refers to the number of DEG annotated to pathways, and the color indicates the significance level of the pathway. The expression of DEG measured by transcriptomic sequencing and real-time reverse-transcription $\mathrm{PCR}(\mathrm{RT}-\mathrm{qPCR} ; \mathrm{C}) . \mathrm{FDR}=$ false discovery rate; $\mathrm{BP}=$ biological process; $\mathrm{CC}=$ cellular component; MF molecular function. Error bars are mean $\pm \mathrm{SD}(\mathrm{n}=3)$. 
JM1_GM001396, and JM1_GM001398), 2 of them encoded sodium-proton antiporters (JM1_GM000185 and JM1_GM001679), and 1 of them encoded alkaline shock proteins (JM1_GM000790). Also, 2 genes encoded choloylglycine hydrolases (JM1_GM000128 and JM1_GM000955), which enhanced the bile salt tolerance of the strain.

\section{Comparative Genomics}

Comparative genomic analysis was conducted to obtain more information of $L$. gasseri JM1. The isolate was highly syntenic with reference strains, and only part $(280,833 \mathrm{bp})$ of the genome sequence was inverted in comparison to L. gasseri ATCC $33323^{\mathrm{T}}$ (Figure 7A). Also, some translocation regions were found between L. gasseri JM1 and K7. As depicted in Figure 7B, the strain shared 1,307 common genes with the reference strains. Notably, 202 unique genes were present in $L$. gasseri JM1 but absent in the reference strains. In the phylogenetic tree constructed based on the single-copy core genes, the genetic relation of JM1 was located close to 6 other strains of L. gasseri species (Figure 7C).

\section{DISCUSSION}

Tolerance to the harsh conditions of gastrointestinal tract, where acid and bile are secreted, is the basic

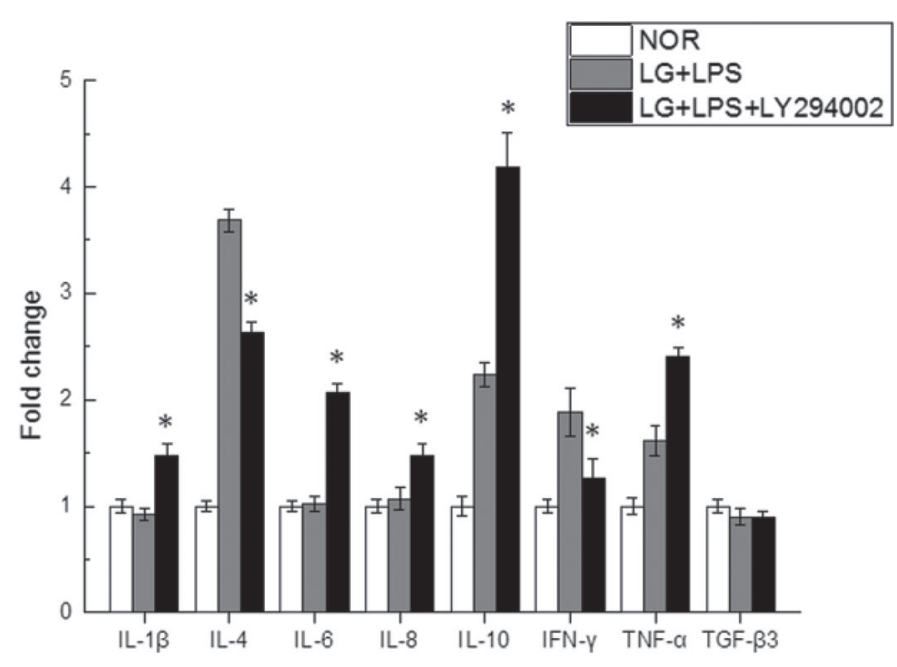

Figure 5. Effect of phosphatidylinositol 3-kinase (PI3K)/protein kinase B (Akt) pathway inhibitor on the expression of cytokines in LPS-stimulated Caco-2 cells. Normal control group (NOR) was treated with Dulbecco's modified Eagle medium alone for $8 \mathrm{~h}$. Caco-2 cells were treated with $1.0 \times 10^{8} \mathrm{cfu} / \mathrm{mL}$ of Lactobacillus gasseri JM1 in the presence (LG+LPS+LY294002) or not (LG+LPS) of PI3K/protein kinase B (Akt) pathway inhibitor for $6 \mathrm{~h}$, and then stimulated with LPS for another $2 \mathrm{~h}$. Data are presented as mean $\pm \mathrm{SD}$ in triplicate. ${ }^{*} P<$ 0.05 was regarded as statistically significant. TNF- $\alpha=$ tumor necrosis factor $\alpha$; TGF- $\beta 3=$ transforming growth factor $\beta 3$. principle for probiotics to exert their potential effects (Huang and Adams, 2004). Lactobacillus gasseri JM1 exhibited high survival percentages of 96.06 and $85.93 \%$ after $3 \mathrm{~h}$ incubation at $\mathrm{pH} 3.0$ and $4 \mathrm{~h}$ cultivation at $0.3 \%$ of bile salts, respectively (Supplemental Tables S1 and S2; https://doi.org/10.3168/jds.2020-18187). Nallala et al. (2017) confirmed that the tolerance to low pH and bile salts of lactic acid bacteria derived from feces is higher than that in fermented dairy products. Some researchers ascribed such bile salt tolerance to the bile salt hydrolase, which could be produced by probiotic strains and break down conjugated bile salts (Bao et al., 2010; Guo et al., 2015). A similar conclusion was obtained in this study because L. gasseri JM1 contained 2 genes encoding choloylglycine hydrolases. Moreover, adhesion to epithelium is an essential prerequisite for probiotics to colonize and compete with pathogenic bacteria in the intestinal tract (Argyri et al., 2013; Lee et al., 2015). With Caco-2 cells serving as an in vitro model, the isolate expressed strong adhesion (7.56 log $\mathrm{cfu} / \mathrm{mL}$ ), suggesting $L$. gasseri JM1 was able to adhere to intestinal epithelial cells and confer benefits to the host. It was noted that the genome encoded fibronectinbinding protein (JM1_GM001105), which was regarded as an adhesion factor. Researchers have revealed that the pili of $E$. coli facilitate adherence to intestinal epithelial cells by identifying specific receptors (Isaacson et al., 1978). Hence, the relatively lower adhesion ability of $E$. coli ATCC 25922 may be associated with the lack of specific receptors in Caco-2 cells. Disorder of intestinal microorganisms can cause gastrointestinal diseases such as diarrhea. In this study, we found that L. gasseri JM1 possessed the ability to inhibit the adhesion of pathogenic bacteria, which was in agreement with the previous report by Bogovic Matijasić et al. (2006) that L. gasseri K7 has significantly inhibited the adhesion of E. coli O8:K88 to Caco-2 cells. In general, lactic acid bacteria protect the host against pathogenic bacteria infection by steric hindrance on the receptors, as well as production of organic acids and bacteriocins (Neeser et al., 2000; Ueda et al., 2011).

Inflammation is a kind of defense response of the innate immunity, which is able to maintain the health of the host by removing harmful pathogens and promote self-repair. Lipopolysaccharide, as an endotoxin component in the cell wall of gram-negative bacteria, can lead to intestinal inflammation and destroy the barrier of epithelial cells (Audy et al., 2012). A previous report showed that LPS substantially triggered the high expression of proinflammatory cytokines, such as TNF- $\alpha$, IL-1 $\beta$, and IL-8 (Jang et al., 2013). Similar observations were found in our study that the production of proinflammatory cytokines (IL-1 $\beta$, IL-6, IL-8, and TNF- $\alpha$ ) was increased significantly in Caco-2 

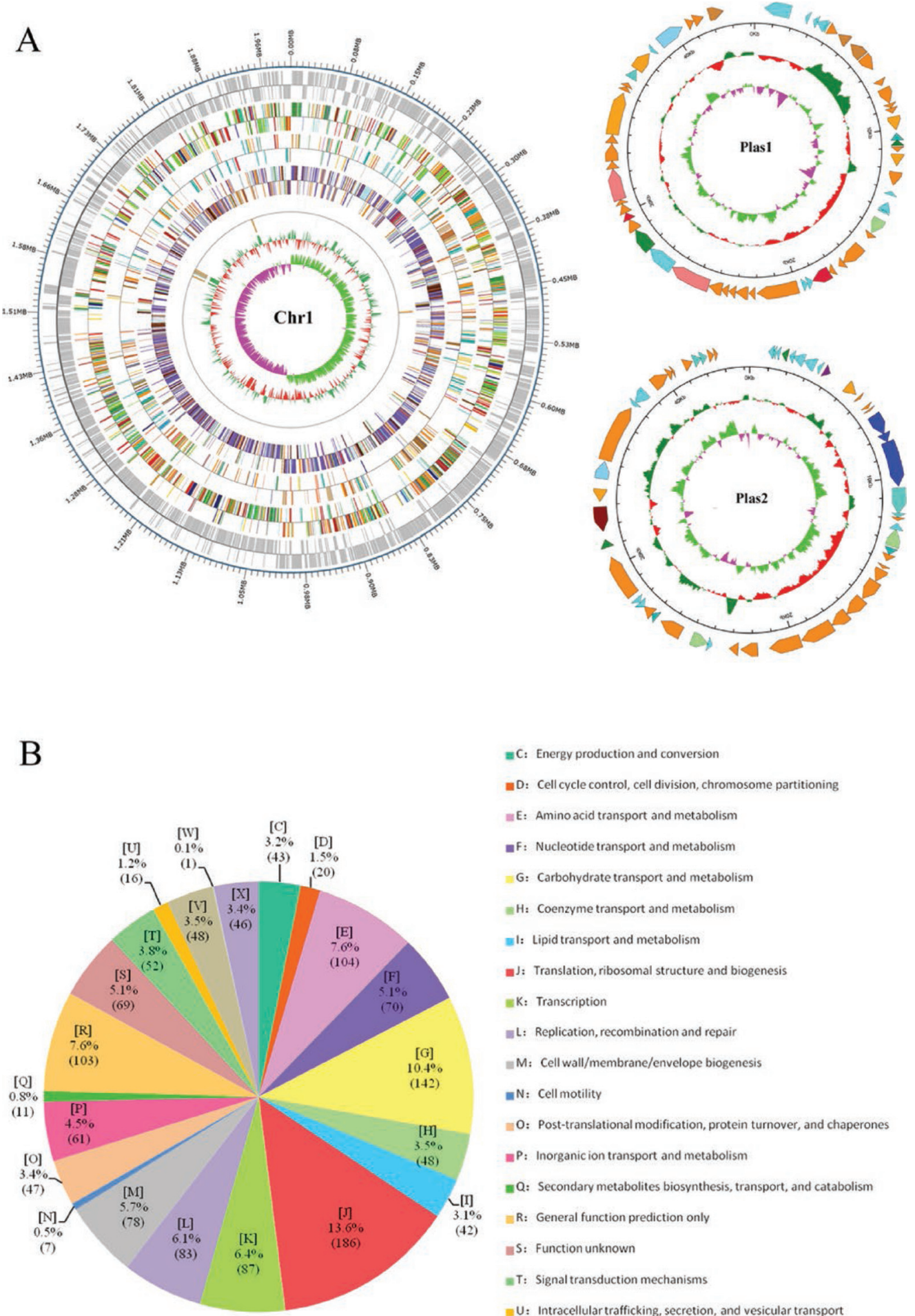

$\equiv \mathrm{C}:$ Energy production and conversion

=D: Cell cycle control, cell division, chromosome partitioning

EE: Amino acid transport and metabolism

mF: Nucleotide transport and metabolism

G: Carbohydrate transport and metabolism

$\approx \mathrm{H}:$ Coenzyme transport and metabolism

I: Lipid transport and metabolism

1n: Translation, ribosomal structure and biogenesis

E: Transcription

wL: Replication, recombination and repair

\# M: Cell wall/membrane/envelope biogenesis

$=\mathrm{N}$ : Cell motility

0: Post-translational modification, protein turnover, and chaperones

-P: Inorganicion transport and metabolism

Q: Secondary metabolites biosynthesis, transport, and catabolism

\# $\mathrm{R}$ : General function prediction only

In: Function unknown

$=\mathrm{T}$ : Signal transduction mechanisms

=U: Intracellular trafficking, secretion, and vesicular transport

EV: Defense mechanism

=W: Extracellular structures

E: Mobilome: prophages, transposons

Figure 6. Genome characteristics of Lactobacillus gasseri JM1. Circular map of L. gasseri JM1 genome (A). From the outermost circle: circle 1 depicts the location of the genome. Circle 2 indicates coding genes. Circles 3, 4, and 5 show the functional annotation of coding genes based on Cluster of Orthologous Groups (COG), Kyoto Encyclopedia of Genes and Genomes, and Gene Ontology databases in order. Circle 6 represents noncoding RNA. Circles 7 and 8 are $\mathrm{G}$ and $\mathrm{C}$ content and $\mathrm{G}-\mathrm{C}$ skew $(\mathrm{G}+\mathrm{C} / \mathrm{G}-\mathrm{C})$, respectively. The $\mathrm{COG}$ functional categories of coding proteins in L. gasseri JM1 genome (B). Chr1 represents the chromosome of L. gasseri JM1. Plas1 and Plas2 are 2 plasmids of the strain. 
A
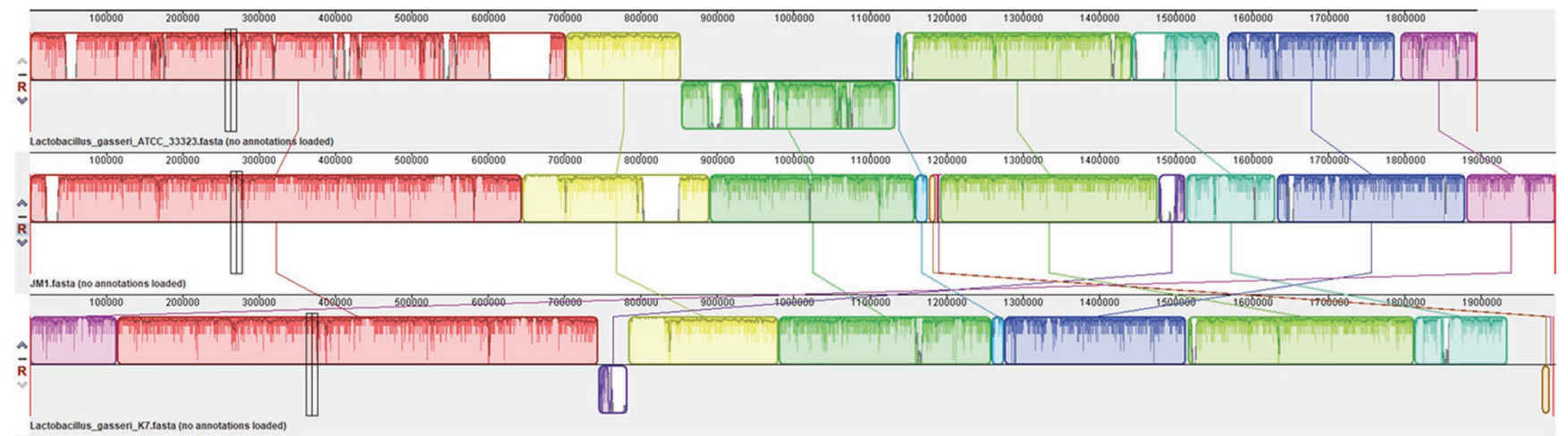

$\mathrm{B}$

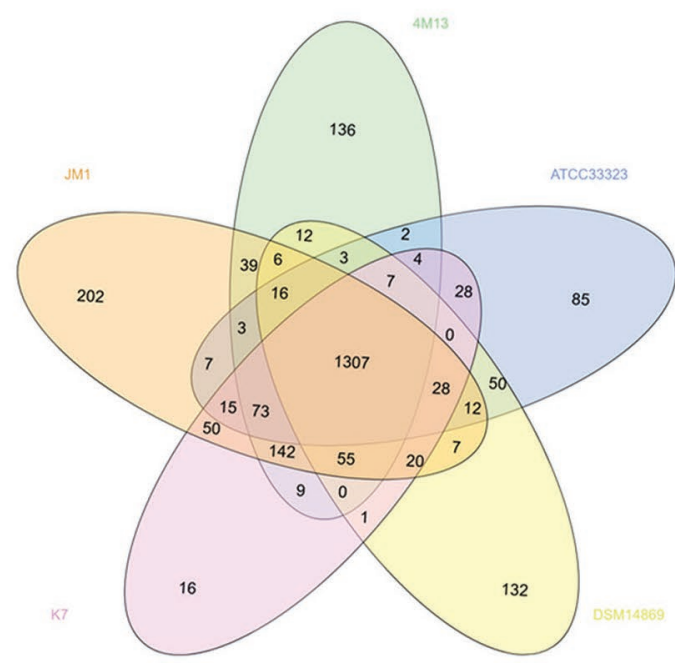

$\mathrm{C}$

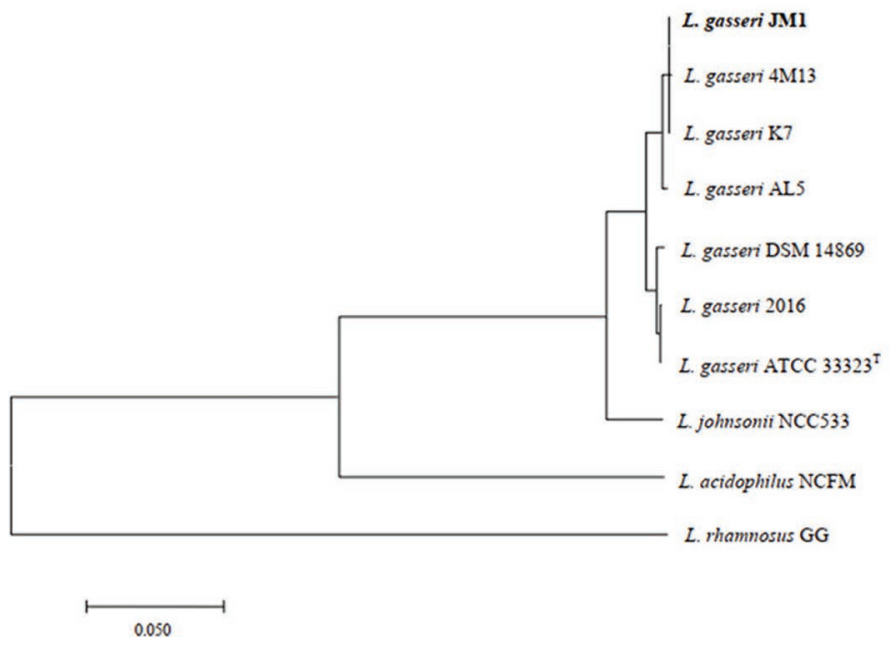

Figure 7. Comparative genomics of Lactobacillus gasseri JM1 with reference strains. Graphical representation of synteny among $L$. gasseri JM1, ATCC $33323^{\mathrm{T}}$, and K7 (A). Venn diagram representing the core-pan analysis of L. gasseri JM1 and 4 reference strains (B). The phylogenetic tree of L. gasseri JM1 with 9 reference strains based on the single-copy core genes (C).

cells. However, pretreatment of L. gasseri JM1 ameliorated the inflammatory response induced by LPS, with downregulation of proinflammatory cytokines and upregulation of anti-inflammatory cytokines (IL-4, IL10 , IFN- $\gamma$, and TGF- $\beta 3$ ). Anti-inflammatory cytokines such as IL-4 and IFN- $\gamma$ were demonstrated to play a crucial role in B lymphocyte differentiation and also in inhibiting Th-2 type immune response (Machura et al., 2007), suggesting the immunoregulatory potential of the strain against LPS stress. Recently, probiotics have been considered as an option for relieving inflammation. In a double-blinded, randomized, control comparative study, the daily consumption of dairy product containing L. gasseri CECT 5714 for 3 mo, in allergic children, enhanced innate and specific immune parameters and induced a significant increase of the lactobacilli counts in feces (Martínez-Cañavate et al., 2009). Similarly, Lactobacillus paraplantarum MTCC 9483 exhibited the anti-inflammatory property under LPS conditions where IL4 and ILI0 mRNA expression levels were upregulated (Devi et al., 2018). In addition, it was noted that our results are consistent with $L$. gasseri $4 \mathrm{M} 13$, a strain isolated from infant feces, except for IL-10, indicating that immunoregulatory effects and mechanisms of probiotics are highly strain specific (Oh et al., 2018).

The intestinal mucosal system as the first line of immunity plays important roles in sensing and responding to environmental stimuli (Kugelberg, 2013). The PRR in mucosa can activate immune response by recognizing and combining with pathogen-associated molecular patterns, linking innate and adaptive immunity. Research on PRR has focused not only on their function in immunity but also on intestinal barrier protection (Cario, 2008), particularly TLR and NLR. A complex relationship exists between TLR and NLR; they complement and synergize in induction of signaling pathways and expression of cytokines (Fukata et al., 2009). A previous study has reported that stress-induced injury was apparent in TLR2-deficient mice, and the activation of 
the TLR2-mediated PI3K/Akt pathway promoted cell survival and maintained integrity of the intestinal epithelial barrier (Oppong et al., 2013). Several Lactobacillus species are able to activate downstream signaling pathways by interacting with intestinal epithelial cells through TLR and NLR cooperatively. Phosphatidylinositol 3-kinase is an intracellular protein with catalytic activity, which can regulate the phosphorylation of downstream Akt and constitute the PI3K/Akt signaling transduction pathway. This pathway is involved in cell proliferation, differentiation, metabolism, and so on (Ciuffreda et al., 2014). Meanwhile, PI3K/Akt is also regarded as a significant immune signaling pathway and interacts with diseases such as angiocardiopathy, tumor, and diabetes. A clinical study on small intestinal adenocarcinoma showed that the PI3K/ Akt signaling pathway was overexpressed compared with normal tissues, indicating this pathway could be deemed a potential therapeutic target (Zhang et al., 2013). Moreover, PI3K/Akt was associated with cell apoptosis and inhibited adhesion of E. coli on epithelial cells by reducing the expression of decay accelerating factor (Banadakoppa et al., 2014). Additional research on Lactobacillus revealed that the combination of $L$. fermentum L930BB and Bifidobacterium animalis ssp. animalis IM386 contributed to intestinal epithelial cell reconstitution and gut homeostasis through the TLR2mediated PI3K/Akt pathway (Paveljšek et al., 2018). In the current study, KEGG analysis revealed that DEG were significantly enriched into the PI3K/Akt pathway. The treatment of inhibitors played various roles in the secretion of cytokines, suggesting the PI3K/Akt pathway took part in the regulation of immunomodulatory activity. In summary, L. gasseri JM1 possessed characteristics to be applied as a probiotic candidate, which include resistance to the harsh conditions, adhesion to intestinal epithelial cells, inhibition of $E$. coli, as well as anti-inflammatory potential. To elucidate the underlying mechanisms, we investigated the expression of PRR and inferred that the strain exerted immunoregulatory effects via the TLR2 and NOD2-mediated PI3K/Akt signaling pathway.

Furthermore, whole-genome sequencing of L. gasseri JM1 was determined to gain insight into genetic properties, and comparative genomic analysis was performed between the isolate and 4 other strains of $L$. gasseri species. Acid stress proteins such $\mathrm{F}_{0} \mathrm{~F}_{1}$-ATPase and sodium-proton antiporters in L. gasseri JM1 can contribute to maintain the homeostasis between intracellular and extracellular pH (Jia et al., 2017). Overall, the presence of acid and bile salt stress proteins encoded in the genome indicates the potential ability of the strain to survive in the gastrointestinal tract.
Genome sequencing also revealed some functional genes involved in the resistance to temperature and oxidative stress, and various stress-related encoding genes such as cold shock protein (JM1_GM001348), heat shock protein (JM1_GM000147, JM1_GM000862), NADH oxidase (JM1_GM001160), pyruvate oxidase (JM1_GM002101), and thioredoxin reductase (JM1_GM001478). The study has demonstrated that temperature-related shock proteins lead to an enhanced capacity in cold adaptation and alleviating the denaturation of intracellular components (Cuthbert et al., 2018). The NADH oxidase, as a kind of oxidoreductase that catalyzes the oxidation reaction of $\mathrm{NADH}$ and $\mathrm{O}_{2}$, has protective effects against oxidative stress in cells (Chen et al., 2014). Moreover, the genomic analysis revealed the presence of immune-related genes including ltaS, tagD, trxA, and dltC. Importantly, the genome encoded 3 gene clusters (JM1_GM000623JM1_GM000640, JM1_GM001955-JM1_GM001967, and JM1_GM002030-JM1_GM002041) for bacteriocin biosynthesis. The results indicated that L. gasseri JM1 possessed the capacity to resist oxidative stress and synthesize bacteriocin, which was in accordance with previous reports (Zorič Peternel et al., 2010; Maldonado-Barragán et al., 2016; Hu et al., 2018). Compared with other L. gasseri strains, the genetic relationship of L. gasseri JM1 with 4M13 and K7 is closer, which may associate to the origin. Lactobacillus gasseri $4 \mathrm{M} 13$ and K7 were isolated from infant feces, the same as JM1, whereas L. gasseri ATCC $33323^{\mathrm{T}}$ and DSM 14869 were strains of human origin. Lactobacillus johnsonii NCC 533 , its closest relative in other species, was located in the neighboring branch. Among the 202 strain-specific genes, 39 were grouped into 16 functional categories based on the COG database. In particular, the high number of coding genes was identified as being involved in "Replication, recombination and repair (L)," "General function prediction only (R)," and "Function unknown (S)." Thus, the function of unique genes needs more detailed investigation.

\section{CONCLUSIONS}

Our results revealed that L. gasseri JM1 could activate the TLR2 and NOD2-mediated PI3K/Akt signaling pathway in intestinal epithelial cells and further alleviate inflammation under LPS conditions by modulation of cytokines. Additionally, whole-genome information confirmed the probiotic properties of L. gasseri JM1 and was helpful for a comprehensive insight into potential characteristics. From the investigation here, L. gasseri JM1, a novel strain with anti-inflammatory effects, could be regarded as a potential probiotic. 


\section{ACKNOWLEDGMENTS}

This work was supported by the National Natural Science Foundation of China (31871828). The authors declare that they do not have any commercial or associative interest that represents a conflict of interest in connection with the work submitted.

\section{REFERENCES}

Ardui, S., A. Ameur, J. R. Vermeesch, and M. S. Hestand. 2018. Single molecule real-time (SMRT) sequencing comes of age: Applications and utilities for medical diagnostics. Nucleic Acids Res. 46:2159 2168. https://doi.org/10.1093/nar/gky066.

Argyri, A. A., G. Zoumpopoulou, K. A. Karatzas, E. Tsakalidou, G. J. Nychas, E. Z. Panagou, and C. C. Tassou. 2013. Selection of potential probiotic lactic acid bacteria from fermented olives by in vitro tests. Food Microbiol. 33:282-291. https://doi.org/10.1016/ j.fm.2012.10.005.

Audy, J., O. Mathieu, J. Belvis, and T. A. Tompkins. 2012. Transcriptomic response of immune signalling pathways in intestinal epithelial cells exposed to lipopolysaccharides, Gram-negative bacteria or potentially probiotic microbes. Benef. Microbes 3:273-286. https://doi.org/10.3920/BM2012.0027.

Azcarate-Peril, M. A., E. Altermann, Y. J. Goh, R. Tallon, R. B. Sanozky-Dawes, E. A. Pfeiler, S. O'Flaherty, B. L. Buck, A. Dobson, T. Duong, M. J. Miller, R. Barrangou, and T. R. Klaenhammer. 2008. Analysis of the genome sequence of Lactobacillus gasseri ATCC 33323 reveals the molecular basis of an autochthonous intestinal organism. Appl. Environ. Microbiol. 74:4610-4625. https://doi.org/10.1128/AEM.00054-08.

Banadakoppa, M., P. Goluszko, D. Liebenthal, B. J. Nowicki, S. Nowicki, and C. Yallampalli. 2014. PI3K/Akt pathway restricts epithelial adhesion of $\mathrm{Dr}+$ Escherichia coli by down-regulating the expression of decay accelerating factor. Exp. Biol. Med. (Maywood) 239:581-594. https://doi.org/10.1177/1535370214522183.

Bao, Y., Y. Zhang, Y. Zhang, Y. Liu, S. Wang, X. Dong, Y. Wang, and H. Zhang. 2010. Screening of potential probiotic properties of Lactobacillus fermentum isolated from traditional dairy products. Food Control 21:695-701. https://doi.org/10.1016/j.foodcont.2009 .10 .010 .

Bogovic Matijasić, B., M. Narat, M. Zorič Peternel, and I. Rogelj. 2006. Ability of Lactobacillus gasseri K 7 to inhibit Escherichia coli adhesion in vitro on Caco-2 cells and ex vivo on pigs' jejunal tissue. Int. J. Food Microbiol. 107:92-96. https://doi.org/10.1016/ j.ijfoodmicro.2005.08.017.

Cario, E. 2008. Barrier-protective function of intestinal epithelial Tolllike receptor 2. Mucosal Immunol. 1(Suppl 1):S62-S66. https://doi .org/10.1038/mi.2008.47.

Chen, X., S. Li, and L. Liu. 2014. Engineering redox balance through cofactor systems. Trends Biotechnol. 32:337-343. https://doi.org/ 10.1016/j.tibtech.2014.04.003.

Ciuffreda, L., U. C. Incani, L. S. Steelman, S. L. Abrams, I. Falcone, A. Del Curatolo, W. H. Chappell, R. A. Franklin, S. Vari, F. Cognetti, J. A. McCubrey, and M. Milella. 2014. Signaling intermediates (MAPK and PI3K) as therapeutic targets in NSCLC. Curr. Pharm. Des. 20:3944-3957. https://doi.org/10.2174/ 13816128113196660763.

Costea, P. I., F. Hildebrand, M. Arumugam, F. Backhed, M. J. Blaser, F. D. Bushman, W. M. de Vos, S. D. Ehrlich, C. M. Fraser, M. Hattori, C. Huttenhower, I. B. Jeffery, D. Knights, J. D. Lewis, R. E. Ley, H. Ochman, P. W. O'Toole, C. Quince, D. A. Relman, F. Shanahan, S. Sunagawa, J. Wang, G. M. Weinstock, G. D. Wu, G. Zeller, L. Zhao, J. Raes, R. Knight, and P. Bork. 2018. Enterotypes in the landscape of gut microbial community composition. Nat. Microbiol. 3:8-16. https://doi.org/10.1038/s41564-017-0072 -8 .
Cuthbert, R. L., R. J. Shute, and D. R. Slivka. 2019. Skeletal muscle cold shock and heat shock protein mRNA response to aerobic exercise in different environmental temperatures. Temperature (Austin) 6:77-84. https://doi.org/10.1080/23328940.2018.1555414.

Dang, F., Y. Jiang, R. Pan, Y. Zhou, S. Wu, R. Wang, K. Zhuang, W. Zhang, T. Li, and C. Man. 2018. Administration of Lactobacillus paracasei ameliorates type 2 diabetes in mice. Food Funct. 9:3630-3639. https://doi.org/10.1039/C8FO00081F.

Devi, S. M., N. K. Kurrey, and P. M. Halami. 2018. In vitro anti-inflammatory activity among probiotic Lactobacillus species isolated from fermented foods. J. Funct. Foods 47:19-27. https://doi.org/ 10.1016/j.jff.2018.05.036.

Dongarrà, M. L., V. Rizzello, L. Muccio, W. Fries, A. Cascio, I. Bonaccorsi, and G. Ferlazzo. 2013. Mucosal immunology and probiotics. Curr. Allergy Asthma Rep. 13:19-26. https://doi.org/10.1007/ s11882-012-0313-0.

Fukata, M., A. S. Vamadevan, and M. T. Abreu. 2009. Toll-like receptors (TLRs) and Nod-like receptors (NLRs) in inflammatory disorders. Semin. Immunol. 21:242-253. https://doi.org/10.1016/ j.smim.2009.06.005.

Galperin, M. Y., K. S. Makarova, Y. I. Wolf, and E. V. Koonin. 2015. Expanded microbial genome coverage and improved protein family annotation in the COG database. Nucleic Acids Res. 43(D1):D261D269. https://doi.org/10.1093/nar/gku1223.

Gao, K., C. Wang, L. Liu, X. Dou, J. Liu, L. Yuan, W. Zhang, and H. Wang. 2017. Immunomodulation and signaling mechanism of Lactobacillus rhamnosus GG and its components on porcine intestinal epithelial cells stimulated by lipopolysaccharide. J. Microbiol. Immunol. Infect. 50:700-713. https://doi.org/10.1016/j.jmii.2015 .05 .002 .

Guo, C.-F., S. Zhang, Y.-H. Yuan, T.-L. Yue, and J.-Y. Li. 2015. Comparison of lactobacilli isolated from Chinese suan-tsai and koumiss for their probiotic and functional properties. J. Funct. Foods 12:294-302. https://doi.org/10.1016/j.jff.2014.11.029.

Hu, J., L. Ma, W. Zheng, Y. Nie, and X. Yan. 2018. Lactobacillus gasseri LA39 activates the oxidative phosphorylation pathway in porcine intestinal epithelial cells. Front. Microbiol. 9:3025-3039. https://doi.org/10.3389/fmicb.2018.03025.

Huang, F. C., and S. C. Huang. 2016. The different effects of probiotics treatment on Salmonella-induced interleukin-8 response in intestinal epithelia cells via PI3K/Akt and NOD2 expression. Benef. Microbes 7:739-748. https://doi.org/10.3920/BM2015.0181.

Huang, Y., and M. C. Adams. 2004. In vitro assessment of the upper gastrointestinal tolerance of potential probiotic dairy propionibacteria. Int. J. Food Microbiol. 91:253-260. https://doi.org/10.1016/ j.ijfoodmicro.2003.07.001.

Isaacson, R. E., P. C. Fusco, C. C. Brinton, and H. W. Moon. 1978. In vitro adhesion of Escherichia coli to porcine small intestinal epithelial cells: Pili as adhesive factors. Infect. Immun. 21:392-397. https://doi.org/10.1128/IAI.21.2.392-397.1978.

Jang, S. E., S. R. Hyam, M. J. Han, S. Y. Kim, B. G. Lee, and D. H. Kim. 2013. Lactobacillus brevis G-101 ameliorates colitis in mice by inhibiting NF- $\kappa \mathrm{B}$, MAPK and AKT pathways and by polarizing M1 macrophages to M2-like macrophages. J. Appl. Microbiol. 115:888-896. https://doi.org/10.1111/jam.12273.

Jia, F. F., L. J. Zhang, X. H. Pang, X. X. Gu, A. Abdelazez, Y. Liang, S. R. Sun, and X. C. Meng. 2017. Complete genome sequence of bacteriocin-producing Lactobacillus plantarum KLDS1.0391, a probiotic strain with gastrointestinal tract resistance and adhesion to the intestinal epithelial cells. Genomics 109:432-437. https:// doi.org/10.1016/j.ygeno.2017.06.008.

Jiang, Y., L. Li, H. Sun, Y. Shan, Y. Liu, L. Li, B. Qu, and C. Man. 2016. Induction of cytokines via NF- $\mathrm{kB}$ and p38 MAP kinase signalling pathways associated with the immunomodulation by Lactobacillus plantarum NDC 75017 in vitro and in vivo. J. Funct. Foods 20:215-225. https://doi.org/10.1016/j.jff.2015.10.027.

Kanehisa, M., G. Susumu, K. Shuichi, O. Yasushi, and H. Masahiro. 2004. The KEGG resource for deciphering the genome. Nucleic Acids Res. 32(suppl 1):277D-280D. https://doi.org/10.1093/nar/ gkh063. 
Kobatake, E., H. Nakagawa, T. Seki, and T. Miyazaki. 2017. Protective effects and functional mechanisms of Lactobacillus gasseri SBT2055 against oxidative stress. PLoS One 12:e0177106. https:/ /doi.org/10.1371/journal.pone.0177106.

Kobayashi, K. S., M. Chamaillard, Y. Ogura, O. Henegariu, and R. A. Flavell. 2005. Nod2-dependent regulation of innate and adaptive immunity in the intestinal tract. Science 307:731-734. https://doi .org/10.1126/science.1104911.

Krzywinski, M., J. Schein, I. Birol, J. Connors, R. Gascoyne, D. Horsman, S. J. Jones, and M. A. Marra. 2009. Circos: An information aesthetic for comparative genomics. Genome Res. 19:1639-1645. https://doi.org/10.1101/gr.092759.109.

Kugelberg, E. 2013. Balancing the gut. Nat. Rev. Immunol. 13:848849. https://doi.org/10.1038/nri3577.

Lee, N.-K., K. J. Han, S.-H. Son, S. J. Eom, S.-K. Lee, and H.-D. Paik. 2015. Multifunctional effect of probiotic Lactococcus lactis KC24 isolated from kimchi. Lebensm. Wiss. Technol. 64:1036-1041. https://doi.org/10.1016/j.lwt.2015.07.019.

Li, L., Y. J. Jiang, X. Y. Yang, Y. Liu, J. Y. Wang, and C. X. Man. 2014. Immunoregulatory effects on Caco-2 cells and mice of exopolysaccharides isolated from Lactobacillus acidophilus NCFM. Food Funct. 5:3261-3268. https://doi.org/10.1039/C4FO00565A.

Lim, D. M., S. Narasimhan, C. Z. Michaylira, and M. L. Wang. 2009. TLR3-mediated NF- $\kappa B$ signaling in human esophageal epithelial cells. Am. J. Physiol. Gastrointest. Liver Physiol. 297:G1172G1180. https://doi.org/10.1152/ajpgi.00065.2009.

Machura, E., B. Mazur, J. Kwiecień, and K. Karczewska. 2007. Intracellular production of IL-2, IL- 4 , IFN- $\gamma$, and TNF- $\alpha$ by peripheral blood CD3 + and CD4 + T cells in children with atopic dermatitis. Eur. J. Pediatr. 166:789-795. https://doi.org/10.1007/s00431 $-006-0319-5$.

Maldonado-Barragán, A., B. Caballero-Guerrero, V. Martin, J. L. Ruiz-Barba, and J. M. Rodriguez. 2016. Purification and genetic characterization of gassericin E, a novel co-culture inducible bacteriocin from Lactobacillus gasseri EV1461 isolated from the vagina of a healthy woman. BMC Microbiol. 16:37-50. https://doi.org/10 .1186/s12866-016-0663-1.

Marin, M. L., J. H. Lee, J. Murtha, Z. Ustunol, and J. J. Pestka. 1997. Differential cytokine production in clonal macrophage and T-cell lines cultured with bifidobacteria. J. Dairy Sci. 80:2713-2720. https://doi.org/10.3168/jds.S0022-0302(97)76232-5.

Martínez-Cañavate, A., S. Sierra, F. Lara-Villoslada, J. Romero, J. Maldonado, J. Boza, J. Xaus, and M. Olivares. 2009. A probiotic dairy product containing L. gasseri CECT5714 and L. coryniformis CECT5711 induces immunological changes in children suffering from allergy. Pediatr. Allergy Immunol. 20:592-600. https:// doi.org/10.1111/j.1399-3038.2008.00833.x.

Mirpuri, J., I. Sotnikov, L. Myers, T. L. Denning, F. Yarovinsky, C. A. Parkos, P. W. Denning, and N. A. Louis. 2012. Lactobacillus rhamnosus (LGG) regulates IL-10 signaling in the developing murine colon through upregulation of the IL-10R2 receptor subunit. PLoS One 7:e51955. https://doi.org/10.1371/journal.pone.0051955.

Nallala, V., V. Sadishkumar, and K. Jeevaratnam. 2017. Molecular characterization of antimicrobial Lactobacillus isolates and evaluation of their probiotic characteristics in vitro for use in poultry. Food Biotechnol. 31:20-41. https://doi.org/10.1080/08905436 .2016.1269289.

Neeser, J. R., D. Granato, M. Rouvet, A. Servin, S. Teneberg, and K. A. Karlsson. 2000. Lactobacillus johnsonii La1 shares carbohydrate-binding specificities with several enteropathogenic bacteria. Glycobiology 10:1193-1199. https://doi.org/10.1093/glycob/10.11 .1193 .

Niibo, M., B. Shirouchi, M. Umegatani, Y. Morita, A. Ogawa, F. Sakai, Y. Kadooka, and M. Sato. 2019. Probiotic Lactobacillus gasseri SBT2055 improves insulin secretion in a diabetic rat model. J. Dairy Sci. 102:997-1006. https://doi.org/10.3168/jds.2018-15203.

Oh, N. S., J. Y. Joung, J. Y. Lee, and Y. Kim. 2018. Probiotic and anti-inflammatory potential of Lactobacillus rhamnosus 4B15 and Lactobacillus gasseri 4M13 isolated from infant feces. PLoS One 13:e0192021. https://doi.org/10.1371/journal.pone.0192021.
Oppong, G. O., G. J. Rapsinski, T. N. Newman, J. H. Nishimori, S. G. Biesecker, and C. Tukel. 2013. Epithelial cells augment barrier function via activation of the Toll-like receptor $2 /$ phosphatidylinositol 3-kinase pathway upon recognition of Salmonella enterica serovar Typhimurium curli fibrils in the gut. Infect. Immun. 81:478-486. https://doi.org/10.1128/IAI.00453-12.

Paveljšek, D., P. Juvan, R. Kosir, D. Rozman, B. Hacin, K. IvicakKocjan, and I. Rogelj. 2018. Lactobacillus fermentum L930BB and Bifidobacterium animalis ssp. animalis IM386 initiate signalling pathways involved in intestinal epithelial barrier protection. Benef. Microbes 9:515-525. https://doi.org/10.3920/BM2017.0107.

Pérez-Cano, F. J., H. Dong, and P. Yaqoob. 2010. In vitro immunomodulatory activity of Lactobacillus fermentum CECT5716 and Lactobacillus salivarius CECT5713: Two probiotic strains isolated from human breast milk. Immunobiology 215:996-1004. https:// doi.org/10.1016/j.imbio.2010.01.004

Powell, S., D. Szklarczyk, K. Trachana, A. Roth, M. Kuhn, J. Muller, R. Arnold, T. Rattei, I. Letunic, T. Doerks, L. J. Jensen, C. von Mering, and P. Bork. 2012. eggNOG v3.0: Orthologous groups covering 1133 organisms at 41 different taxonomic ranges. Nucleic Acids Res. 40(D1):D284-D289. https://doi.org/10.1093/nar/ gkr1060.

Ren, D., C. Li, Y. Qin, R. Yin, X. Li, M. Tian, S. Du, H. Guo, C. Liu, N. Zhu, D. Sun, Y. Li, and N. Jin. 2012. Inhibition of Staphylococcus aureus adherence to Caco- 2 cells by lactobacilli and cell surface properties that influence attachment. Anaerobe 18:508-515. https: //doi.org/10.1016/j.anaerobe.2012.08.001.

Sakamoto, I., M. Igarashi, K. Kimura, A. Takagi, T. Miwa, and Y. Koga. 2001. Suppressive effect of Lactobacillus gasseri OLL 2716 (LG21) on Helicobacter pylori infection in humans. J. Antimicrob. Chemother. 47:709-710. https://doi.org/10.1093/jac/47.5.709.

Selle, K., and T. R. Klaenhammer. 2013. Genomic and phenotypic evidence for probiotic influences of Lactobacillus gasseri on human health. FEMS Microbiol. Rev. 37:915-935. https://doi.org/10 .1111/1574-6976.12021.

Sommer, F., and F. Backhed. 2013. The gut microbiota-masters of host development and physiology. Nat. Rev. Microbiol. 11:227-238. https://doi.org/10.1038/nrmicro2974.

Ueda, S., M. Kinoshita, F. Tanaka, M. Tsuboi, S. Shimizu, N. Oohata, M. Hino, M. Yamada, Y. Isogai, and S. Hashimoto. 2011. Strain selection and scale-up fermentation for FR901379 acylase production by Streptomyces sp. no. 6907. J. Biosci. Bioeng. 112:409-414. https://doi.org/10.1016/j.jbiosc.2011.06.002.

Yamamoto, M., and S. Akira. 2005. Mechanisms of innate immune responses mediated by Toll-like receptors. Clin. Appl. Immunol. Rev. 5:167-183. https://doi.org/10.1016/j.cair.2005.02.001.

Zhang, H. J., J. M. Qu, C. Z. Shao, J. Zhang, L. X. He, and Z. H. Yuan. 2008. Aspergillus fumigatus conidia upregulates NOD2 protein expression both in vitro and in vivo. Acta Pharmacol. Sin 29:1202-1208. https://doi.org/10.1111/j.1745-7254.2008.00860.x.

Zhang, Y., Y. Xue, J. Congqing, Y. Junqiu, G. Jing, C. Honglei, H. Mohammed, W. Yan, and F. Lifang. 2013. Expression of PI3K, PTEN and Akt in small intestinal adenocarcinoma detected by quantum dots-based immunofluorescence technology. Cancer Biomark. 13:299-305. https://doi.org/10.3233/CBM-130352.

Zorič Peternel, M., A. Canzek Majhenic, H. Holo, I. F. Nes, Z. Salehian, A. Berlec, and I. Rogelj. 2010. Wide-inhibitory spectra bacteriocins produced by Lactobacillus gasseri K7. Probiotics Antimicrob. Proteins 2:233-240. https://doi.org/10.1007/s12602-010 -9044-5.

\section{ORCIDS}

Xinyu Liu (® https://orcid.org/0000-0002-7182-239X Xuesong Li @ \ttps://orcid.org/0000-0002-6884-4627 Chaoxin Man (1) https://orcid.org/0000-0003-2050-4639 Yujun Jiang (i) https://orcid.org/0000-0002-2857-2137 